\title{
Information reduction in a reverberatory neuronal network through convergence to complex oscillatory firing patterns
}

\author{
A. Vidybida ${ }^{\mathrm{a}, *}$, O. Shchur ${ }^{\mathrm{b}}$ \\ ${ }^{a}$ Bogolyubov Institute for Theoretical Physics, Metrologichna str., 14-B, Kyiv 03680, \\ Ukraine \\ ${ }^{b}$ Taras Shevchenko National University of Kyiv, Volodymyrska str., 60, Kyiv 01033, Ukraine
}

\begin{abstract}
We study dynamics of a reverberating neural net by means of computer simulation. The net, which is composed of 9 leaky integrate-and-fire (LIF) neurons arranged in a square lattice, is fully connected with interneuronal communication delay proportional to the corresponding distance. The network is initially stimulated with different stimuli and then goes freely. For each stimulus, in the course of free evolution, activity either dies out completely or the network converges to a periodic trajectory, which may be different for different stimuli. The latter is observed for a set of 285290 initial stimuli which constitutes $83 \%$ of all stimuli applied. By applying each stimulus from the set, we found 102 different periodic end-states. By analyzing the trajectories, we conclude that neuronal firing is the necessary prerequisite for merging different trajectories into a single one, which eventually transforms into a periodic regime. Observed phenomena of self-organization in the time domain are discussed as a possible model for processes taking place during perception. The repetitive firing in the periodic regimes could underpin memory formation.
\end{abstract}

Keywords: reverberating network, periodic regime, attractor, stability, signal processing, perception

\section{Introduction}

Recordings from the inferior temporal cortex (IT) have revealed cells with unusual properties compared with cells in the primary visual cortex, [25, 34, 15]. Firstly, those cells have extremely extended receptive fields. Secondly, those cells are selective for complex features/objects, such as complex shapes. Thirdly, this

\footnotetext{
* Corresponding author

Email addresses: vidybida@bitp.kiev.ua (A. Vidybida), olha.schur@gmail.com (O. Shchur)

URL: http://vidybida.kiev.ua/ (A. Vidybida)
}

Preprint submitted to Elsevier 
selectivity is invariant with respect to transformations, such as changing of object's position in space, its orientation, size, color, texture, contrast, location on the retina, $[36,17]$. Furthermore, the response of those cells is stable with changing context and the presence of distractors (tolerance to noise). It looks like primary visual signals are stripped of redundant information during processing in the visual pathways $[6,4,17]$. It is interesting that similar redundancy stripping is characteristic of sensory pathways of other modalities, such as hearing [14] and olfaction $[41,16]$ as well as in the primary visual areas, see review in $[21]$.

At the sensory periphery, information about external world contains all data which primary sensory units, such as cones and rods, or olfactory receptor neurons are able to sense and transmit further. In a sense, representation at the sensory periphery may be considered as continuous, [30]. On the other hand, in farther areas of the brain, like IT, or multimodal areas, where recognition and perception take place, representation of the real world is conceptual and as such has a binary, discrete nature. This process of transformation of sensory inputs from continuous, redundant representation to binary conceptual representation accompanied with and enabled by the redundancy reduction is sometimes called as information condensation, [30]. Specifically in vision, the term "dimension reduction" is as well used, [17].

We now propose a question: What might be the physical mechanism of the information condensation? The visual perception is fast: $50 \mathrm{~ms}$ period of image presentation is enough to form corresponding perception state 100-150 ms later. It is hard to expect structural changes in the neuronal network during that short period. If we believe together with H. Barlow [5] and F. Crick [13] that "the activities of neurons, quite simply, are thought processes", then we must expect that it is the spatiotemporal pattern of neuronal activity which changes during perception process and eventually forms a state of perception of the object presented to sensory periphery.

A possible mechanism of a proper change was proposed by D. MacKay as self-organization in the time domain [31]. Due to self-organization, the spatiotemporal dynamics of neural activity in a pulse-propagating network is able to develop the same regime in response to several different initial stimuli which could be treated as redundancy reduction. This mechanism was checked in [38] for a network composed of 5 binding neurons. It was found that that simple network can have several hundreds of different periodic regimes, each being the result of stimulation by any stimulus from the corresponding set. Obtaining the same final regime in response to different stimuli means that corresponding dynamical trajectories converge and finally merge in the time course and this might be that process which makes definite cells in the IT insensitive to certain differences in the primary visual stimuli.

In the theory of mechanical dynamical systems, convergence of trajectories is a well known phenomenon that is a consequence of mechanical/viscous friction. Systems of this type can be described by differential equations amenable to standard stability analysis. In a pulse propagating neuronal network, neuronal leakage is the analog of friction in a mechanical system. This is because 
due to the leakage the electrical energy is dissipated to heat similarly as does mechanical energy due to mechanical friction. But the dynamics of spiking neurons is not smooth, which makes these systems unsuitable for standard stability analysis. This may account for the relative paucity of studies of these systems. Among those studies, a network of spiking neurons considered in $[28,29]$ has zero interneuronal communication delays. The net is composed of neurons without leakage and is in the presence of constant and permanent external excitatory stimulation to each neuron. In [22], a similar system is analyzed, but finite communication delays are taken into account. In spite of visible limitations, like usage of a single stimulus, namely a constant excitatory input to each unit, interesting conclusions are made in [28, 29, 22]. Among those is the existence of many periodic regimes, which are reached after single triggering of each neuron.

The purpose of this investigation is to figure out possible elementary physical processes and events underpinning convergence of different dynamical trajectories, evoked by different initial stimulation of a reverberating neural net, with eventual merging them into a single one and further engaging in a periodic regime.

In the context of perception, we pay a special attention to periodic regimes for the following reason. An effective perceptual operation involves the ability to report out what has been perceived, see e.g. [4]. This is in contrast to reception, where the fastest reaction might bring the best result, e.g. [11]. The ability to report supposes that perceived object is held in a short-term memory of some sort. This is in concordance with the finding of several types of memory in the IT, see [32]. The short-term memory through long-term potentiation requires repetitive stimulation of the same synapses,[3], and this can be well achieved during periodic dynamics in the neuronal network.

Indirect experimental evidence that some neuronal assemblies can be engaged into a periodic-like regime is vast, $[7,37,10]$. Among those are stimulusevoked oscillation-type activities, see [18, 19, 24, 20]. Activities of single neurons are observed to be phase-locked with each other, but not with the stimulus presentation moment. This suggests that those activities have a self-organized, emergent nature. Similar suggestions might be derived from observation of socalled unitary events in the motor cortex during preparation and execution of movements, see [33, 27, 26].

\section{Methods}

\subsection{Neural network}

For performing simulations we use a network of nine excitatory leaky integrateand-fire (LIF) neurons organized into a square lattice of definite geometric size, Fig.1. The interneuronal communication delays are calculated based on the interneuronal distance and the communication speed. Each neuron is characterized by the same firing threshold, $V_{0}$, the relaxation time, $\tau$ and the unitary EPSP height, $h$, see legend to Fig.1,2 for the numerical values. The network is fully connected and each neuron has additional external excitatory input used 


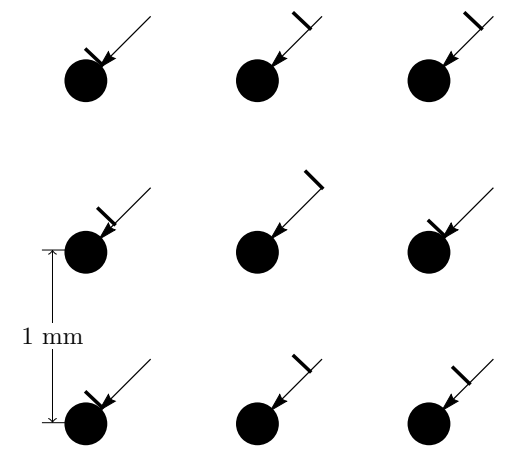

Figure 1: Network used in simulations. The network is fully connected (the internal connections are not shown). The interneuronal communication speed $v=1 \mathrm{~m} / \mathrm{s}$. The communication delay between neurons with the positions $(i, j)$ and $(k, l)$, is calculated based on the Euclidean distance: $d_{(i, j),(k, l)}=1 \mathrm{~mm} \cdot \sqrt{(i-k)^{2}+(j-l)^{2}} / v$. Each input impulse got from internal connection rises the LIF's excitation level by $2.71 \mathrm{mV}$. The internal synaptic weights, $W_{(i, j),(k, l)}=1$ for all $i, j, k, l=0,1,2,(i, j) \neq(k, l)$. Impulse received from an external connection (shown by arrows) rises excitation just above the firing threshold. This corresponds to the input synaptic weights $W_{(i, j)}^{\text {input }} \geq 7.39$.

for delivering initial stimulation necessary to start reverberating dynamics. Example of a single stimulus for the whole network is given in Figs. 1,3.
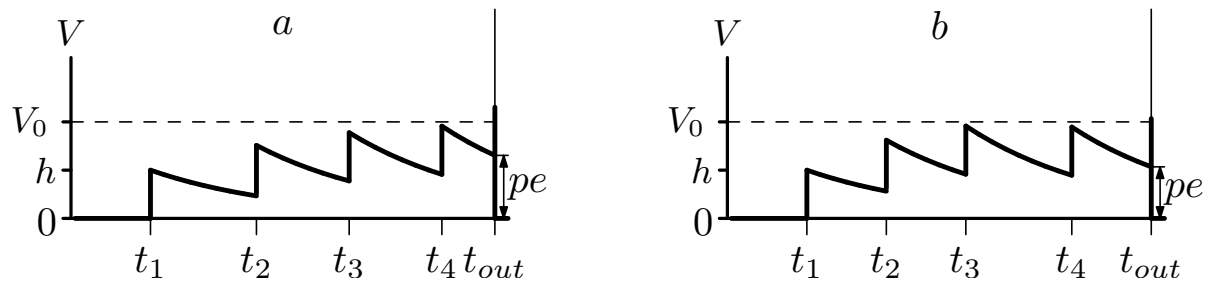

Figure 2: Example of two different stimuli into a single LIF neuron, which evoke identical outputs. Each neuron has a firing threshold $V_{0}=20 \mathrm{mV}$, The relaxation time of any LIF neuron $\tau=20 \mathrm{~ms}$. The triggering input spike is delivered at time $t_{\text {out }}$. Both stimuli evoke the same output (a spike at the same moment) in spite of having a different temporal position of input spikes \#2-\#4. The different timing results in different pre-triggering excitation, pe in the figures. The pe difference is cancelled after triggering.

The network states during evolution are generated with the time step $d t=$ $0.1 \mathrm{~ms}$. Interneuronal communication delays, which do not equal to a whole number of $d t$, are rounded, e.g. delay through the diagonal is taken $2.8 \mathrm{~ms}$ instead of $10 \sqrt{8} d t$. The LIF states are expressed in terms of integers as well, see Sec. 2.1.1 below. This allows us to run evolution by means of integer arithmetics and to figure out exact periodic regimes of the net. 


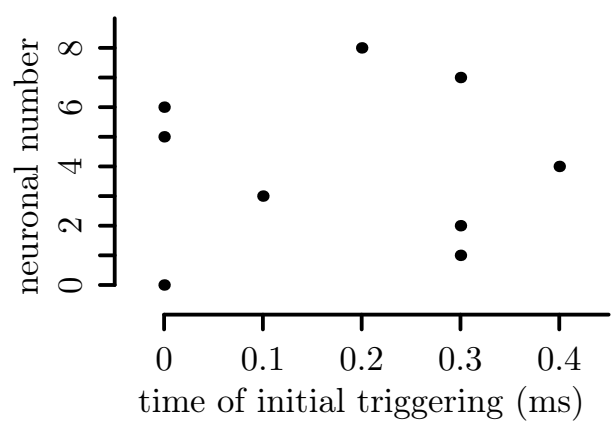

Figure 3: Example of a single stimulus to start a reverberating dynamics. Moments of initial triggering shown here exactly correspond to positions of bars in Fig. 1. Each dot specifies the triggering time of corresponding neuron (vertical axis) due to the stimulus. Neuron \#0 is always triggered at the moment when a stimulus starts.

\subsubsection{Simulating LIF neuron with integers}

Dynamics of a spiking neural network can be unstable. This is because in some dynamical states infinitely small delay in arriving moment of input triggering spike may prevent neuron from achieving the firing threshold at that moment. Then the neuronal triggering will be initiated by the next or further input spike. As a result, the triggering time will change considerably and this change can be amplified during further evolution. Instability requires that, in the search of a periodic regime, states are reproduced exactly after the regime's period. On the other hand, exact equality cannot be stated in computation with floating-point numbers, see [23] for discussion.

In order to overcome this difficulty, an approximation method has been developed, [40]. In this method, the whole set of LIF states, namely the interval of real numbers $\left[0 ; V_{0}\right]$, is approximated by a finite set of discrete values unevenly distributed in the $\left[0 ; V_{0}\right]$. Each discrete value is labeled by a pair of integers $(n, i)$. Either receiving of input impulse or evolving during simulation time step $d t$ results in changing the pair. This approximates evolution of LIF states expressed in terms of floating point numbers by the evolution of states expressed in terms of integer labels and makes them amenable for exact comparison in computer. The two models are denoted as fpLIF and intLIF. The quality of such approximation is checked by generating triggering moments of both fpLIF and intLIF under fairly long random inputs. It was established that with suitable choice of approximation parameters the sequences of firing moments of both models match with each other exactly if expressed in terms of a whole number of $d t$. See [40] for details.

\subsection{Data acquisition}

The purpose of running the simulation was to characterize the input-out behavior of the network, i.e. to enumerate all periodic regimes to which the network evolves given an initial stimulus taken from a definite set S. We mean 
here that the network can settle into some periodic firing pattern that was not necessarily related in any simple way to the stimulus. This periodic firing pattern is attained due to self-organization in the time domain. Many different initial stimuli may result in the same periodic pattern. The set of stimuli has elements $\left\{t_{0}=0, t_{1}, \ldots, t_{8}\right\}$, where $t_{i}$ denotes the moment (in $d t$ units) of triggering of neuron \# $i$ due to the stimulus, see Fig. 3. Each $t_{i}$ can take any value from $\{0,1,2,3,4\}$ except $t_{0}$, which is always 0 . We leave initial firing moment for the neuron \#0 invariable in order to eliminate degeneration due to the uniform shift of initial firing moments for all neurons. Thus, the first stimulus in the set $\mathrm{S}$ is $\{0,0,0,0,0,0,0,0,0\}$ and the last one is $\{0,4,4,4,4,4,4,4,4\}$ with total number of stimuli $5^{8}=390625$. For each individual stimulus, we consider the moment of arrival of last input triggering spike from that stimulus as $t=0$ for the subsequent net's evolution.

After applying a stimulus from S, the net's evolution had been running with time step $d t$ without external stimulation until a state identical to already observed in this run occurred. Since the dynamics is deterministic, the occurrence means that a periodic regime has been achieved. The entire sequence of states covering the periodic regime is enclosed between the first and the second occurrence of the two identical states, one of them included. The result of each run was stored in two Berkeley Database tables. The first table, IniNum.db, has the initial stimulus as its first column. The second column is composed as a pair of corresponding periodic regime serial number and relaxation time required to entrain on that regime after the end of the initial stimulation. For some stimuli dynamic decays to the dead state with no triggering. This state was numbered as 0 . The second table, NumPesta.db, has a representative of the periodic regime as its first column. Actually, this was exactly the repeated state mentioned above. The second column is the regime's serial number. Kicked with two different stimuli, the net may entrain onto the same regime in two different points of the cycle, see Fig. $6(\mathrm{C})$. In order not to count these cases as two different regimes, we have performed an adequate checking.

For observing the convergence progress during approaching the same cycle, we have generated the whole set of trajectories corresponding to that cycle and then have analyzed them with additionally developed programs.

\section{Results}

\subsection{Periodic regimes and their domains}

After trying all stimuli from the set $\mathrm{S}$, we have obtained 102 different periodic regimes. This splits S into $102+1$ disjoint subsets corresponding to different periodic regimes ${ }^{1}$. "1" stays for the dead regime. The histogram of sizes of the

\footnotetext{
${ }^{1}$ In paper [38] for a network of 5 neurons it was found much more, namely 485 different periodic regimes. This is because in [38] moments of initial triggering could take 100 different values which gives for the size of set $\mathrm{S}$ as much as 100000000 stimuli instead of 390625 as in this work. Extending the set $\mathrm{S}$ might bring about more periodic regimes for our network. This is expected to check in future work.
} 


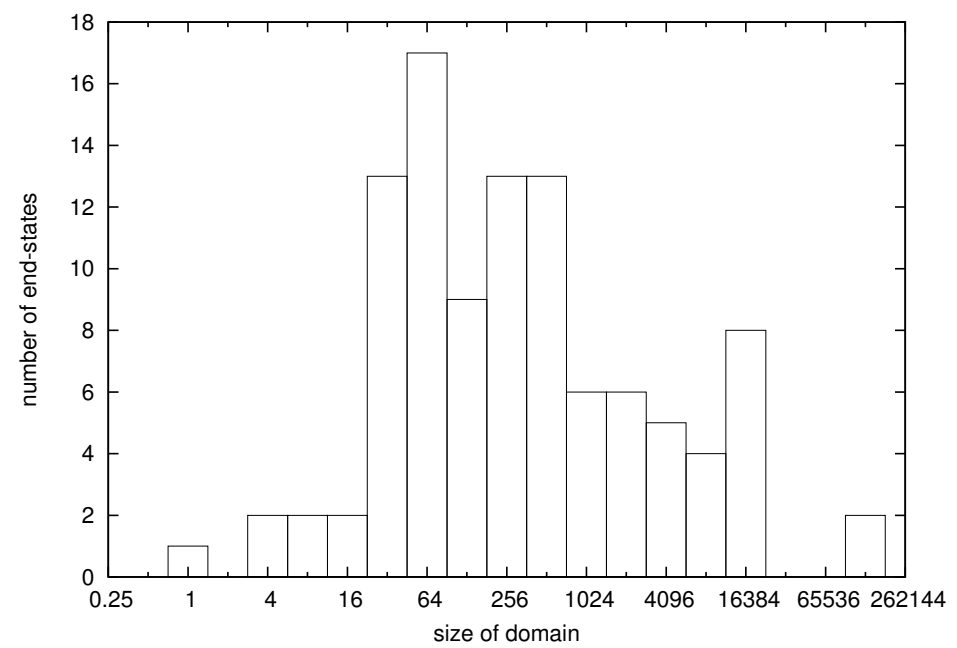

Figure 4: Histogram of sizes of attractor domain. This is for 285290 stimuli giving rise to one of the 102 periodic end-states. Another 105335 stimuli giving rise to the dead end-state are not presented here.

\begin{tabular}{|c|c|c|c|c|c|c|c|c|}
\hline period (ms) & 3.0 & 3.2 & 3.4 & 3.6 & 4.0 & 6.8 & 7.2 & 10.4 \\
\hline number of regimes & 5 & 20 & 11 & 29 & 19 & 6 & 6 & 6 \\
\hline
\end{tabular}

Table 1: Number of regimes with a given period.

subsets is given in Fig.4.

In the theory of dynamical systems, the term "attractor basin" is used. The basin is composed of all points in the phase space of a system, which approach corresponding attractor due to dynamic. Here we consider not phase points (each phase point is composed of states of all individual neurons and axons) but points in the set of stimuli (each point is composed of the moments of delivering initial triggering stimulus for each neuron). Therefore, we use for the above subsets the term "attractor domain" instead of "attractor basin".

Periods of the regimes found are given in Table 1.

For a given periodic regime, each of the 9 neurons fires the same number of spikes during the whole cycle. This number depends on the period's length as shown in Table 2.

\subsection{Routes to periodic regime}

Before the dynamics enter into the periodic regime, each neuron has been triggered 1 to 10 times. The relaxation times required to enter the periodic regimes are shown in Fig. 5. In some cases, all trajectories belonging to a single 


\begin{tabular}{|c|c|c|c|}
\hline spikes per period & 1 & 2 & 3 \\
\hline period duration (ms) & $3.0,3.2,3.4,3.6,4.0$ & $6.8,7.2$ & 10.4 \\
\hline
\end{tabular}

Table 2: Number of spikes per period.

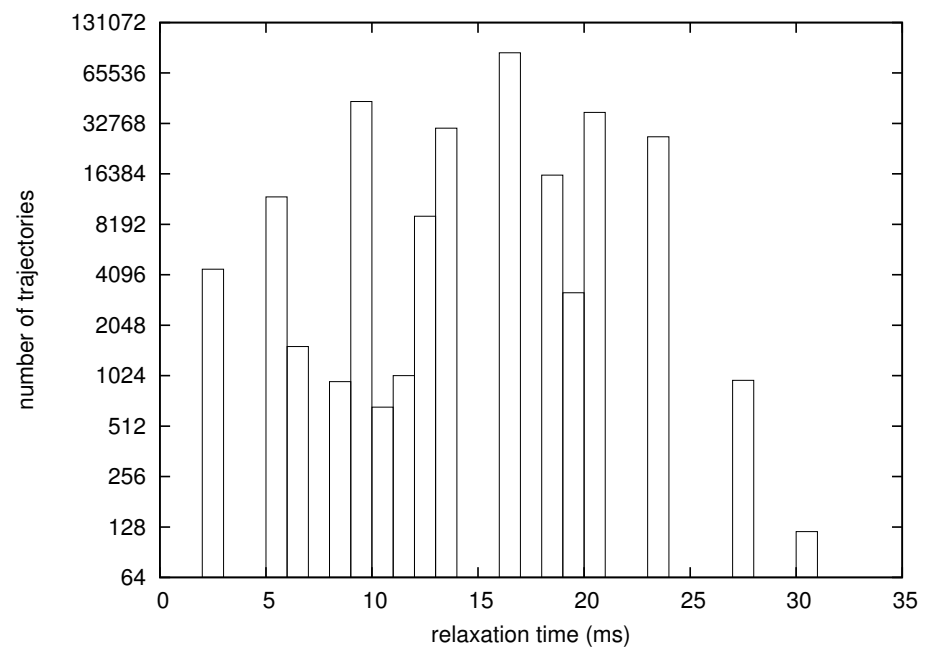

Figure 5: Histogram of relaxation times required for attaining a periodic regime.

periodic regime have the same duration, in other cases the trajectories may have several different duration.

Several possible routes for the system to evolve into a periodic regime were observed. Three examples are given in Fig. 6. The first one is realized if all trajectories merge into a single one well before the periodic regime is reached, see Fig. 6, (A). The second one is realized if all trajectories merge into a smaller number of different trajectories, which evolve some time before entering the cycle at the same point, see Fig. 6, (B). The third one is realized if all trajectories enter the cycle at two different points, see Fig. 6, (C).

Two states were considered different in the analysis if the state of any axon or neural element did not match. Therefore, synchronous firing of all corresponding neurons in two different trajectories still does not imply that the trajectories are already merged. The same is valid for establishing periodicity: a reproduced pattern of firing still does not guarantee that a periodic regime is attained. An example of the latter can be observed in Fig. 7.

\subsection{Triggering is necessary for merging}

In order to test the working hypothesis, all trajectories corresponding to the 102 periodic end-states were saved into separate files. A program has been de- 
A

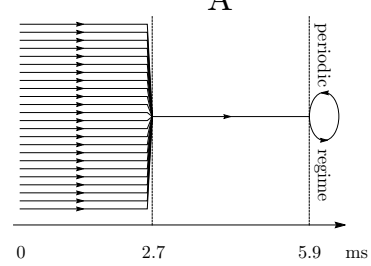

$\mathrm{B}$

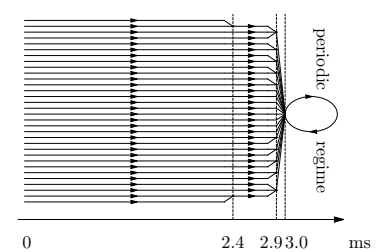

$\mathrm{C}$

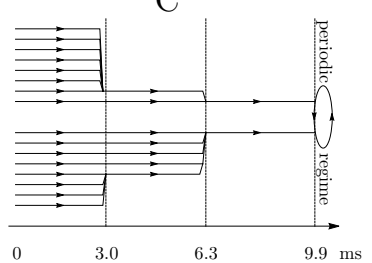

Figure 6: Routes to common periodic regime. (A) - state \#83; (B) - state \#34; (C) - state \#96, not all trajectories are shown.

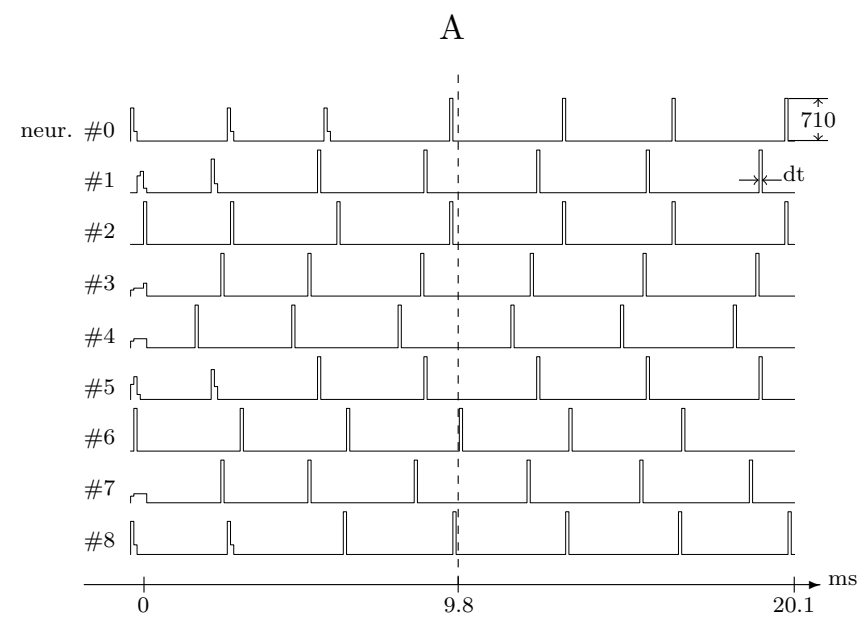

B

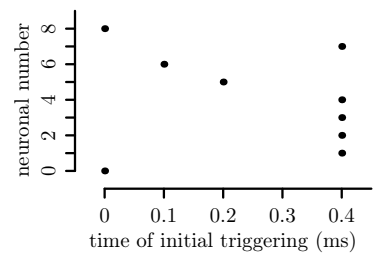

C

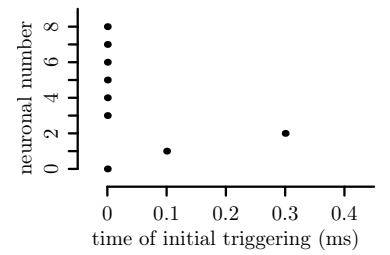

$\mathrm{D}$

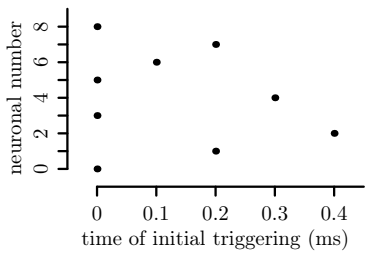

Figure 7: (A) Spiking moments of the neurons at all trajectories corresponding to a single domain. The domain for this regime (\#6) includes 710 different stimuli. The periodic regime starts after $9.8 \mathrm{~ms}$ and has period $10.4 \mathrm{~ms}$. (B)-(D) - examples of the initial stimuli delivering this regime. Notice that the moment $t=0$ in (A) corresponds to the last triggering moment in the stimulus, namely, to $t=0.4 \mathrm{~ms}$ in (B), (D), and to $t=0.3 \mathrm{~ms}$ in (C). 


\begin{tabular}{|c|c|c|c|}
\hline voltage perturbation size & $1 \%$ & $0.5 \%$ & $0.1 \%$ \\
\hline stable & 10 & 47 & 81 \\
\hline unstable, total & 92 & 55 & 21 \\
\hline $\begin{array}{l}\text { unstable due to approaching another } \\
\text { known periodic regime }\end{array}$ & 36 & 35 & 20 \\
\hline $\begin{array}{l}\text { unstable due to approaching another } \\
\text { unknown periodic regime }\end{array}$ & 7 & 1 & 0 \\
\hline $\begin{array}{l}\text { unstable due to approaching the dead } \\
\text { state }\end{array}$ & 49 & 19 & 1 \\
\hline
\end{tabular}

Table 3: Result of checking stability with respect to perturbation of neuronal voltage.

veloped which analyzes what has happened just before two or more trajectories merge into a single one. The program was applied to trajectory sets corresponding to each periodic regime. The output of the program has shown us that at the moment of merging, one or more neurons in the net is triggered. Also, triggering was observed, which was not followed by merging. This leads to a conclusion that triggering is the necessary prerequisite for merging.

\subsection{Stability}

Finally, the robustness of the periodic end-states was tested. The numerical simulation is performed in whole numbers. Therefore we can use only finite perturbation of a state in order to check its stability. The state of our network is described by the values of time to live of impulses in all axons as well as excitation level in each neuron. We have checked separately the stability with respect to axonal and neuronal perturbations of all 102 periodic regimes found. In order to perturb a single neuron's state we calculated the excitation voltage $V$ corresponding to state variables $(n, i)$ (see Sec. 2.1.1 above) and changed its value by up to $\pm 1 \%$. The new $(n, i)$ pair was found, corresponding to the new $V$ and used as a new (perturbed) neuronal state. After that the net was allowed to execute a free running until a periodic state or the dead state was achieved. If the achieved periodic regime was the same as used for perturbation, and this takes place for perturbation of any of 9 neurons the network is composed of and for all states the cycle is composed of, the unperturbed regime was considered as stable with respect to neuronal perturbation. The obtained results are given in Tab. 3 . The smallest possible perturbation of neuronal state can be achieved if in the $(n, i)$ pair the $i$ value is replaced with $i \pm 1$. This corresponds to perturbation of physical voltage by $2.49 \cdot 10^{-10 \%}$ to $2.51 \cdot 10^{-10 \%}$. Under perturbation of this kind, all 102 periodic states have appeared to be stable. 


\begin{tabular}{|c|c|}
\hline \multicolumn{1}{|c|}{ axonal perturbation size } & $d t=0.1 \mathrm{~ms}$ \\
\hline \multicolumn{1}{|c|}{ stable } & 31 \\
\hline $\begin{array}{l}\text { unstable, total } \\
\text { known periodic regime }\end{array}$ & 71 \\
\hline $\begin{array}{l}\text { unstable due to approaching another } \\
\text { unknown periodic regime }\end{array}$ & 83 \\
\hline $\begin{array}{l}\text { unstable due to approaching the dead } \\
\text { state }\end{array}$ & 20 \\
\hline
\end{tabular}

Table 4: Result of checking stability with respect to perturbation of axonal states. Here, the time to live of impulse in axon was perturbed by one $d t$.

In order to check stability with respect to axonal perturbation, we have perturbed the time to live of an impulse (if any) in the axon by $\pm d t$. If the time to live was just equal to the axonal delay, then only $-d t$ perturbation was used. If the time to live was zero, then only $+d t$ was used. We have proceeded further similarly as described above for neuronal states perturbation. The result is shown in Tab. 4.

We also have tried the same system with the same set of stimuli, but with the LIF relaxation time $\tau=200 \mathrm{~ms}$ instead of $20 \mathrm{~ms}$. A set of 67 different periodic end-states have been obtained with periods 3.0, 3.2, 3.4, 3.6, 4.8, 6.6. $7.2,10.4 \mathrm{~ms}$, which is rather close to what is listed in the Tab. 1 . This might be expected since possible period values are restricted by the values of propagation times, which were the same.

\section{Discussion and Conclusions}

Dynamics is investigated of a pulse-propagating neural net propelled into reverberating evolution by different initial stimuli. Apart from final end-states in which all spiking activity was extinguished, two self-organized behaviors were observed: 1) merging of several dynamical trajectories into a single common trajectory; 2) entrainment of several initially different trajectories into a single, common periodic regime.

At the beginning of this study, it was expected that convergence/merging of different trajectories happens due to neuronal firing. This idea is briefly discussed in [38, Sec. 2.1.1] for the binding neuron model. Firing of a single neuron happens at the moment of receiving the last (triggering) input impulse. Positions of impulses, which precede the triggering one, may deviate slightly 
without changing the triggering moment, see Fig. 2. This way, two different input stimuli into a neuron are merged into a single output spike emitted at the same moment of time. Due to consecutive merging in neurons the network is composed of, two different dynamics may converge and eventually merge into a single one. The results obtained confirm that spiking is necessary for the merging of trajectories in this simulation. This conclusion is made by strict analysis of the trajectory files just before merging and after that. In all merging cases, we have observed that some neurons have a bit different subthreshold excitation at different trajectories. These neurons were simultaneously triggered before merging and that small difference in excitation was forgotten. For a single neuron, this mechanism is illustrated in Fig. 2.

In the signal/information-processing context, neuronal firing results in emitting a spike, which can be treated as an abstract representation of a set of input spikes into the neuron [39]. Due to cascade of such abstractions, several different trajectories can merge into a single one, which can be treated as removing redundancy present in the initial stimuli. This same mechanism can be responsible for the noise tolerance observed during perception, [5, 4]. Final attainment of a periodic regime might be treated as an effect underpinning perception, see Fig. 8, since periodic/repetitive firing results in formation of the short-term memory due to long-term potentiation [3]. A kind of memory is believed to be required for perception, [4].

\begin{tabular}{|l|l}
$\begin{array}{l}\text { single neuron firing - } \\
\text { abstraction in a single } \\
\text { neuron }\end{array}$
\end{tabular}$\Rightarrow \begin{aligned} & \begin{array}{l}\text { merging of trajectories } \\
\text { - redundancy stripping }\end{array}\end{aligned} \Rightarrow$\begin{tabular}{l}
$\begin{array}{l}\text { periodic regime - final } \\
\text { abstraction and memo- } \\
\text { rization }\end{array}$ \\
\hline
\end{tabular}

Figure 8: A possible route to perception as an emergent phenomenon due to self-organization in the time domain.

The simplified neuronal dynamics and signal processing assumptions embodied in the simulation have some obvious limitations:

1. The system relies on recurrent connections and reverberation rather than feed-forward dynamics. While the recurrence does have a role in perception, see, e.g. [9, 8] for vision and [35] for hearing, the feed-forward mode of processing is definitely present. For a discussion of the feed-forward mode see [1, 2]. Both modes can be combined, see, e.g. [42].

2.The number of neural elements is small $(n=9)$. Actually, it is established for the IT, [25], and expected for other senses, [5], that objects are presented by activity of small neuronal sets. May it be as small as 9? A further investigation is required with a larger network.

3. Certainly, real neural networks, unlike these simulations, do not reproduce a periodic regime forever. In reality, one should expect a regime close to periodic, which is eventually interrupted by a mechanism not considered here.

4. The only structural change we mention here is at the final stage of processing, namely the long-term potentiation due to periodic regime. It is possible that faster, spike timing dependent facilitations and inhibitions might play a role in neural dynamics even on short time scales. A possible fast mechanism is the 
twitching of dendritic spines, [12].

At the same time, we see that dynamics of a reverberating neural network can be rich with different regimes emerging due to spatio-temporal self-organization. This endows reverberating networks with sophisticated abilities to process signals/information. These remain to be further investigated.

Acknowledgments. AV thanks to Demiurge Technologies AG for support with attendance of several neuroscience conferences.

\section{References}

[1] M. Abeles. Local Cortical Circuits: An Electrophysiological study. Springer, 1982.

[2] A. Aertsen, M. Diesmann, and M. O. Gewaltig. Propagating of synchronous spiking activity in feedforward neural networks. J.Physiol.(Paris), 90:243247, 1996.

[3] P. Andersen. A prelude to long-term potentiation. Philosophical Transactions of the Royal Society B: Biological Sciences, 358(1432):613-615, 2003.

[4] H. Barlow. Hidden agenda: A sceptical view of the privacy of perception. In Perception and the Physical World, pages 305-316. John Wiley \& Sons, Ltd, 2002.

[5] H. Barlow. Single units and sensation: A neuron doctrine for perceptual psychology? Perception, pages 371-394, 1972.

[6] H. Barlow. Possible principles underlying the transformations of sensory messages. In W. Rosenblith, editor, Sensory Communication, pages 217234. M.I.T. Press, 1961.

[7] E. Başar. Memory and brain dynamics : oscillations integrating attention, perception, learning, and memory. CRC Press, 2004.

[8] J. Bullier. Feedback connections and conscious vision. Trends in Cognitive.Sciences., 5(9):369-370, 2001.

[9] J. Bullier. Integrated model of visual processing. Brain Research.Reviews., 36(2-3):96-107, 2001.

[10] G. Buzsáki. Rhythms of the brain. Oxford University Press, 2006.

[11] J. M. Camhi and W. Tom. The escape behavior of the cockroach Periplaneta americana. Journal of comparative physiology., 128(3):193-201, 1978.

[12] F. Crick. Do dendritic spines twitch? Trends Neurosci., 5(2):44-46, 1982.

[13] F. Crick. The Astonishing Hypothesis. Touchstone, 1995. 
[14] O, Creutzfeldt, F.-C. Hellweg and Chr. Schreiner. Thalamocortical transformation of responses to complex auditory stimuli. Experimental Brain Research., 39(1):87-104, 1980.

[15] J. J. DiCarlo, D. Zoccolan, and N. C. Rust. How does the brain solve visual object recognition? Neuron, 73(3):415-434, 2012.

[16] P. Duchamp-Viret and A. Duchamp. Odor processing in the frog olfactory system. Prog.Neurobiol., 53:561-602, 1997.

[17] R. Durbin and G. Mitchison. A dimension reduction framework for understanding cortical maps. Nature, 343(6259):644-647, 1990.

[18] R. Eckhorn, R. Bauer, W. Jordan, M. Brosch, W. Kruse, M. Munk, and H. J. Reitboeck. Coherent oscillations: a mechanism for feature linking in the visual cortex? Biological Cybernetics, 60:121-130, 1988.

[19] R. Eckhorn, H. J. Reitboeck, M. Arndt, and P. Dicke. Feature linking via synchronization among distributed assemblies: simulations of results from cat visual cortex. Neural Computation, 2:293-307, 1990.

[20] A. K. Engel, P. König, A. K. Kreiter, C. M. Gray, and W. Singer. Temporal coding by coherent oscillations as a potential solution to the binding problem: physiological evidence. In H. G. Schuster and W. Singer, editors, Nonlinear Dynamics and Neuronal Networks, pages 3-25. VCH Weinheim, 1991.

[21] D. J. Felleman and D. C. Van Essen. Distributed hierarchical processing in the primate cerebral cortex. Cerebral cortex, 1(1):1-47, 1991.

[22] W. Gerstner. Rapid phase locking in systems of pulse-coupled oscillators with delays. Physical Review Letters, 76(10):1755-1758, 1996.

[23] D. Goldberg. What every computer scientist should know about floatingpoint arithmetic. ACM Comput.Surv., 23(1):5-48, 1991.

[24] C. M. Gray, P. König, A. K. Engel, and W. Singer. Oscillatory responses in cat visual cortex exibit intercolumnar synchronization which reflects global stimulus properties. Nature, 338:334, 1989.

[25] C. Gross, R. Desimone, T. Albright, and E. Schwartz. Inferior temporal cortex as a visual integration area. In F. Reinoso-Suarez and C. AjmoneMarsan, editors, Cortical Integration, pages 291-315. Raven Press, 1984.

[26] S. Grün. Data-driven significance estimation for precise spike correlation. Journal of Neurophysiology, 101:1126-1140, 2009.

[27] S. Grün, M. Diesmann, and A. Aertsen. Unitary event analysis. In S. Grün and S. Rotter, editors, Analysis of Parallel Spike Trains, Springer Series in Computational Neuroscience 7, pages 191-220. Springer, 2010. 
[28] A. V. Herz and J. J. Hopfield. Earthquake cycles and neural reverberations: Collective oscillations in systems with pulse-coupled threshold elements. Physical Review Letters, , 75(6):1222-1225, 1995.

[29] J. J. Hopfield and A. V. Herz. Rapid local synchronization of action potentials: toward computation with coupled integrate-and-fire neurons. Proceedings of the National Academy of Sciences of the USA, 92(15):66556662, 1995.

[30] P. König and N. Krüger. Symbols as self-emergent entities in an optimization process of feature extraction and predictions. Biological Cybernetics, 94:325-334, 2006.

[31] D. M. MacKay. Self-organization in the time domain. In M. C. Yovitz, G. T. Jacobi, and G. Goldstein, editors, Self-Organizing Systems, pages 37-48. Spartan Books, 1962.

[32] Y. Miyashita. Inferior temporal cortex: where visual perception meets memory. Annual review of neuroscience, 16:245-263, 1993.

[33] A. Riehle, S. Grün, M. Diesmann, and A. Aertsen. Spike synchronization and rate modulation differentially involved in motor cortical function. Science, 278(5216):1950-1953, 1997.

[34] N. C. Rust and J. J. DiCarlo. Selectivity and tolerance ("invariance") both increase as visual information propagates from cortical area V4 to IT. The Journal of Neuroscience, 30(39):12978-12995, 2010.

[35] J. H. Siegel and D. O. Kim. Efferent neural control of cochlear mechanics? olivocochlear bundle stimulation affects cochlear biomechanical nonlinearity. Hearing Res., 6:171-182, 1982.

[36] S. Tang, R. Wolf, S. Xu, and M. Heisenberg. Visual pattern recognition in Drosophila is invariant for retinal position. Science, 305(5686):1020-1022, 2004.

[37] R. D. Traub, J. G. L. Jefferys, and M. A. Whittington. Fast oscillations in cortical circuits. MIT Press, 1999.

[38] A. K. Vidybida. Testing of information condensation in a model reverberating spiking neural network. International Journal of Neural Systems, 21(3):187-198, 2011.

[39] A. K. Vidybida. Binding neuron. In M. Khosrow-Pour, editor, Encyclopedia of information science and technology, pages 1123-1134. IGI Global, 2014.

[40] A. K. Vidybida. Simulating leaky integrate and fire neuron with integers. Mathematics and Computers in Simulation, (submitted, see also arXiv:1505.06603). 
[41] M. Wehr and G. Laurent. Odor encoding by temporal sequences of firing in oscillating neural assemblies. Nature, 384:162-166, 1996.

[42] P. Zheng and J. Triesch. Robust development of synfire chains from multiple plasticity mechanisms. Frontiers in Computational Neuroscience, 8:66, 2014. doi: $10.3389 /$ fncom.2014.00066 